\title{
Loop Equations in Abelian Gauge Theories
}

\author{
Cayetano Di Bartolo ${ }^{1}$, Lorenzo Leal ${ }^{2}$ and Francisco Peña ${ }^{1,2}$ \\ 1. Departamento de Física, Universidad Simón Bolívar, \\ Aptdo. 89000, Caracas 1080-A, Venezuela. \\ 2. Grupo de Campos y Partículas, \\ Departamento de Física, Facultad de Ciencias, \\ Universidad Central de Venezuela, \\ AP 47270, Caracas 1041-A, Venezuela.
}

\begin{abstract}
The equations obeyed by the vacuum expectation value of the Wilson loop of Abelian gauge theories are considered from the point of view of the loop-space. An approximative scheme for studying these loop-equations for lattice Maxwell theory is presented. The approximation leads to a partial difference equation in the area and length variables of the loop, and certain physically motivated ansatz is seen to reproduce the mean field results from a geometrical perspective.
\end{abstract}

\section{INTRODUCTION}

The loop representation [1, 2, 3, 4, 5] is a useful tool for studying non-perturbative features of gauge theories, both in the lattice and the continuous frameworks. Loop-space formulations of quantum gravity have also been developed, giving rise to a geometrical setting in which some of the long-standing questions concerning the small distance behavior of space-time can be properly addressed $[6,7,8,8,[10]$.

Some years ago, the loop representation formulation of Hamiltonian lattice gauge theories was studied for several models 11, 12, 13, 14]. The goal of that formulation was to produce loop-dependent Schrödinger equations, and to develop loop-based schemes of approximation to study the spectra and the phase-transitions of the theories. The purpose of this paper is to draw some preliminary lines for a similar study within the Lagrangian formulation. Concretely, we shall study the loop-equation that obeys the Wilson loop average $\langle W(C)\rangle$ of Maxwell theory in the lattice. We shall be interested in exploring some basic aspects of the loop content of the theory rather than in obtaining accurate predictions for the relevant observables.

As a warm-up for the lattice study, we first recall briefly some basic facts about loop-equations for Abelian gauge theories in the continuum. As it is well known, the loop-equation [15, 16, 17, 18] (sometimes called Migdal, Polyakov or Schwinger-Dyson equation) for $\langle W(C)\rangle$ can be exactly solved for the free Maxwell theory within the continuous framework [19].

In the non-Abelian cases, loop-equations have been considered mainly within the large $N$ approximation 16, 17, 18, 20]. Some recent applications of the loop-equation approach to QCD can be found in references 21, 22, 23. On the other hand, in the lattice, even the Abelian cases are non-trivial (except in two dimensions), due to the presence of the phase transition that separates the confining regime (absent in the formulation in the continuum) from the weak coupling one.

The paper is organized as follows. In section two we review the Wilson loop equations for Maxwell and ChernSimons theories in the continuum from a loop-space perspective. In the last section we consider Maxwell theory in the lattice.

\section{MAXWELL AND CHERN-SIMONS WILSON-LOOP EQUATIONS IN THE CONTINUUM}

We shall deal with the vacuum expectation value

$$
\langle W(C)\rangle \equiv \int D A W(C) \exp i S,
$$

of the Wilson loop

$$
W(C) \equiv \exp \left(-i e \oint_{C} d x^{\mu} A_{\mu}(x)\right),
$$

where $S$ is the action functional of an Abelian gauge theory, that we shall first take as the n-dimensional Maxwell one

$$
S_{\text {Maxwell }}=-\frac{1}{4} \int d^{n} x F_{\mu \nu} F^{\mu \nu} .
$$


Since $S_{\text {Maxwell }}$ is quadratic in the fields, the functional integration in equation (11) can be performed. Instead, we are interested in studying the functional differential equation that $\langle W(C)\rangle$ obeys, i.e., in the Migdal loop-equation of the model. To this end, we shall use a few tools of the loop-space formulation of gauge theories [1, 2, 3, 4, 5, 29].

Consider the space of oriented and piecewise continuous curves in $R^{n}$. We say that curves $\gamma$ and $\gamma^{\prime}$ are equivalent if they share the same form factor $T^{\mu}(x, \gamma)$, defined as

$$
T^{\mu}(x, \gamma) \equiv \int_{\gamma} d y^{\mu} \delta^{(n)}(\vec{x}-\vec{y})
$$

Every equivalence class defines a path. The composition of curves, together with the equivalence relation stated above, defines a group product among paths. It can be shown that this group is Abelian. Now, let us consider path-dependent functionals $\Psi(\gamma)$. We introduce the path derivative $\delta_{\mu}(x)$, that measures the change in $\Psi(\gamma)$ when an infinitesimal path $u$ is attached to the argument $\gamma$ of $\Psi(\gamma)$ at the point $x$, up to the first order in the vector $u^{\mu}$ associated to the path

$$
\Psi(u \cdot \gamma)=\left(1+u^{\mu} \delta_{\mu}(x)\right) \Psi(\gamma)
$$

We shall also use the loop derivative $\Delta_{\mu \nu}(x)$ defined as

$$
\Psi(\sigma \cdot \gamma)=\left(1+\sigma^{\mu \nu} \Delta_{\mu \nu}(x)\right) \Psi(\gamma)
$$

with $\sigma^{\mu \nu}$ being the area enclosed by an infinitesimal loop $\sigma$ attached at the point $x$. The loop derivative is readily seen to be the curl of the path derivative

$$
\Delta_{\mu \nu}(x)=\partial_{\mu} \delta_{\nu}(x)-\partial_{\nu} \delta_{\mu}(x)
$$

Also, we have

$$
\delta_{\mu}(x) T^{\nu}(y, \gamma)=\delta_{\mu}^{\nu} \delta^{(n)}(x-y)
$$

as can be readily shown.

Since $\langle W(C)\rangle$ is a genuine path-space function [it depends on closed paths $C$ ], it makes sense to take its loop derivative. Using the last equation, it is easy to see that

$$
\Delta_{\mu \nu}(x)\langle W(C)\rangle=-i e \int D A F_{\mu \nu}(x) W(C) \exp \{i S\} .
$$

Taking the divergence of this expression and recalling the variational principle for the Maxwell equations, we find

$$
\partial^{\mu} \Delta_{\mu \nu}(x)\langle W(C)\rangle=e \int D A \frac{\delta W(C)}{\delta A^{\nu}(x)} e^{i S},
$$

where we have also integrated by parts in the functional sense. Using

$$
\frac{\delta W(C)}{\delta A^{\nu}(x)}=-\operatorname{ieg}_{\mu \nu} T^{\mu}(x, C) W(C)
$$

we finally obtain the desired loop-equation

$$
\partial^{\mu} \Delta_{\mu \nu}\langle W(C)\rangle=-i e^{2} g_{\mu \nu} T^{\mu}(x, C)\langle W(C)\rangle .
$$

It should be stressed that everything in equation (12) is a true loop-dependent object, in the sense of the equivalence classes mentioned at the beginning of this section. Equation (12) is a loop-space version of the ordinary differential equation

$$
a \frac{d F}{d x}(x)=x F(x)
$$

whose solution is $F(x)=\exp \left(\frac{x^{2}}{2 a}\right)$. Following this hint, one can readily obtain the solution

$$
\langle W(C)\rangle=K \exp \left[\int d^{n} x \int d^{n} y T^{\mu}(x, C) D_{\mu \nu}(x-y) T^{\nu}(y, C)\right],
$$


where $D_{\mu \nu}(x-y)$ is the Feynman propagator of the theory. Finally

$$
\langle W(C)\rangle=K \exp \left[-\frac{e^{2}}{8 \pi^{2}} \oint_{c} d x^{\mu} \oint_{c} d y^{\nu} \frac{g_{\mu \nu}}{|x-y|^{2}}\right]
$$

as it should be [30].

In the preceding discussion, we ignored that, due to gauge invariance, the Wilson loop average given by equation (11) is ill-defined. To remedy this, one may add the gauge-fixing term $-\frac{1}{2 \xi}\left(\partial_{\mu} A^{\mu}\right)^{2}$ to the Maxwell action (2). In that case, equation (12) changes into

$$
\left[\partial^{\mu} \Delta_{\mu \nu}(x)+\frac{1}{\xi} \partial_{\nu} \partial^{\mu} \delta_{\mu}(x)\right]\langle W(C)\rangle=-i e^{2} g_{\mu \nu} T^{\mu}(x, C)\langle W(C)\rangle,
$$

but the net result (15) does not change.

To conclude this section, let us briefly consider the Chern-Simons model, a topological theory whose action is

$$
S[A]=\int \mathrm{d}^{3} x \varepsilon^{\mu \nu \lambda} A_{\mu} \partial_{\nu} A_{\lambda} .
$$

Now the differential equation for the Wilson loop is

$$
\varepsilon^{\mu \nu \lambda} \Delta_{\nu \lambda}(x)\langle W(C)\rangle=-i e^{2} T^{\mu}(x, C)\langle W(C)\rangle .
$$

It is interesting to notice that, being the model topological, its associated loop-equation is metric independent. This contrasts with what occurs in Maxwell theory.

As before, the solution to the loop-equation should be an exponential quadratic in the "loop-coordinates" $T{ }^{\mu}(x, C)$. In fact, it is given by

$$
\langle W(C)\rangle=\exp \left[-\frac{i e^{2}}{4} G(C, C)\right]
$$

where

$$
G(C, C)=\frac{1}{4 \pi} \oint_{c} d x^{\mu} \oint_{c} d y^{\nu} \varepsilon_{\mu \nu \lambda} \frac{(x-y)^{\lambda}}{|x-y|^{3}}
$$

is the Gauss self-linking number of the loop C, which is a well known knot-invariant. Again, this result coincides with what is obtained by performing the functional integral (1) for the Chern-Simons model [31].

This way of calculating $\langle W(C)\rangle$ can also be used for other Abelian gauge theories, such as the Maxwell-ChernSimons [32] and the Proca model in the Stueckelberg formulation [29], since the differential loop-equations are similar to the Maxwell loop-equation (12).

\section{MAXWELL WILSON-LOOP EQUATION IN THE LATTICE}

Now we turn to consider loop-equations in the lattice. The partition function of compact electrodynamics, which is the model we are going to study, is given by

$$
Z=\int_{0}^{2 \Pi}\left(\prod_{l>0} d \theta_{l}\right) \exp (-S)
$$

with

$$
S=-\beta \sum_{p>0}\left(W(p)+W^{*}(p)\right)
$$

and

$$
W(p)=\prod_{l \in p} \exp \left(i \theta_{l}\right)
$$


The Wilson functional $W(C)$ is defined, as usual, by

$$
W(C)=\prod_{l \in C} \exp \left(i \theta_{l}\right)
$$

In these expressions, $l, p$ and $C$ denote links, plaquettes and closed loops respectively, $\beta$ is the inverse of the temperature and $\theta_{l}$ is the angular variable associated to link $l$.

The Schwinger-Dyson equation for the Wilson loop, that corresponds to the lattice version of equation (12), may be written as 24]

$$
\beta \sum_{p} \Delta(l, p)<W(p \cdot C)>+\Delta(l, C)<W(C)>=0
$$

where

$$
\Delta(l, C) \equiv \sum_{l^{\prime} \in C} \widetilde{\delta}_{l l^{\prime}}
$$

and

$$
\widetilde{\delta}_{l l^{\prime}} \equiv\left\{\begin{aligned}
1 & \text { for } l=l^{\prime} \\
-1 & \text { for } l=\bar{l}^{\prime} \\
0 & \text { otherwise }
\end{aligned}\right.
$$

By $\bar{l}$ we mean the opposite of link $l$, and $<>$ means statistical average. In equation (25) the sum runs over all the plaquettes of the lattice, regardless of their orientation. The factor $\Delta(l, p)$, however, restricts the sum to the plaquettes attached to the link $l$. The loop product $p \cdot C$ must be understood as the yuxtaposition of plaquette $p$ and loop $C$, yielding a new closed loop. This corresponds to the lattice version of the loop-derivative of Gambini-Trí as [1, 4] given in equation (6). Unlike non-Abelian loops, U(1) loops commute (i.e., they are not "ordered") and do not have a marked starting point.

Equation (25) arises by equating the averages of the Wilson loop for two different values of the phase factor $\exp i \theta_{l}$ assigned to a fixed link $l$ of the lattice. This equality is a consequence of the invariance of the measure under $U(1)$ transformations. Expression (25), which indeed represents one equation for each link of the lattice, may be summed over all the links belonging to the loop $C$ to produce the single equation

$$
\beta \sum_{l \in C} \sum_{p} \Delta(l, p)<W(p \cdot C)>+\Lambda_{C}<W(C)>=0,
$$

where

$$
\Lambda_{C} \equiv \sum_{l \in C} \sum_{l^{\prime} \in C} \delta_{l l^{\prime}}=\sum_{i=1}^{\infty} i^{2} L_{i},
$$

with $L_{i}$ being the number of links that appear $i$ times in the loop $C$. For simple loops (i.e., loops without multiple links) $\Lambda_{C}$ coincides with the length of $C$.

Equation (28) (or (25) ) is a loop-equation, since any reference to the electromagnetic potential has been summed when averaging over the different field configurations. Despite its simple appearance (it is linear and first order in the lattice "loop derivative") and unlike its continuous counterpart, it is not possible, as far as we know, to solve it exactly (except for $D=2$ ). We shall discuss a simple method to extract some information of this equation that relies on similar ideas that were applied to the Hamiltonian lattice formulation in the past [1, 13].

To characterize completely a loop one needs an infinite amount of variables. An incomplete list of them could contain the length, area, number and types of corners, the class of knottiness to which the loop belongs, and quite a few other variables. One could select, on physical grounds, some of these variables and ignore the (necessarily infinite number of) remaining ones. Another point of view could be to consider, instead, all the loops up to a given size, which leads to a linked-cluster approach [12]. Here we shall adopt the first point of view, i.e., a "collective variables" approach, which has already been considered to deal with the $Z_{2}$ Hamiltonian gauge theory [11, 13]. To motivate the choice of variables we are going to make, we can reason as follows.

It is well known that for "big" loops and $D>3$, the Wilson functional decreases exponentially with: a) the length, for the weak coupling regime $\left(\beta=1 / g^{2} \rightarrow+\infty\right)$; b) the area, for the strong coupling one $(g$ is the coupling constant, or the square root of the temperature) 25, 26, 27]. For $D=2$, the theory can be solved exactly, and the area 
dependence holds for any value of the coupling constant (i.e., the theory presents a single confining phase). For $D=3$, approximation schemes and numerical simulations yield that the situation seems to be very similar to the $D>3$ cases, although the exact solution has not been found [25, 26, 27]. All this suggests that length and area are good candidates to describe, in a first approximation, the Wilson loop functional. Moreover, the length variables $L_{i}$ already appear explicitly in the loop-equation (28). With this guide in mind, we approximate $\langle W(C)>$ in the form

$$
<W(C)>\approx f\left(L_{1}, L_{2}, \cdots ; A_{1}, A_{2}, \cdots\right) \quad,
$$

where $A_{i}$ is the number of plaquettes of multiplicity $i$ appearing in the loop $C$.

This guess about the possible relevant loop-variables should be accompanied by some restrictions on the loops that we are going to take into account. Since we are not including "corners" in the list of variables, it seems reasonable to ask our loops to be composed of lengthy straight paths, which could be formed by multiple links. Furthermore, to avoid ambiguities about how the first term in equation (28) modifies the area variables, we must restrict ourselves to consider planar loops.

We are ready to write down equation (28) for $f\left(L_{i} ; A_{i}\right)$. The first term in this equation produces several types of contributions, depending on how the plaquette $p$ is appended to the loop $C$. Let us begin with

$$
+(2 D-3) i L_{i}\left(f\left(L_{1}+3, L_{i}-1, L_{i+1}+1 ; A_{1}+1\right)-f\left(L_{1}+3, L_{i}-1, L_{i-1}+1 ; A_{1}+1\right),\right.
$$

that corresponds to taking into account all the plaquettes that hit the loop $C$ at links of type $i$, in such a way that these plaquettes lie outside the area of the loop. The first term in this expression arises when the links of the plaquette and the loop that make contact have the same orientation. In the second term, the links mentioned above have opposite orientations. We have omitted the dependence of $f$ in the variables which are not modified by the attachment of the plaquette.

The second contribution is given by

$$
i L_{i}\left(f\left(L_{1}+3, L_{i}-1, L_{i+1}+1 ; A_{i}-1, A_{i+1}+1\right)-f\left(L_{1}+3, L_{i}-1, L_{i-1}+1 ; A_{i}-1, A_{i-1}+1\right)\right),
$$

and is produced by plaquettes that lie on the loop area.

Putting all the contributions together, one arrives to

$$
\begin{aligned}
\sum_{i=0}^{\infty} i L_{i}\left\{\frac{i}{\beta} f+\right. & f\left(L_{1}+3, L_{i}-1, L_{i+1}+1 ; A_{i}-1, A_{i+1}+1\right)-f\left(L_{1}+3, L_{i}-1, L_{i-1}+1 ; A_{i}-1, A_{i-1}+1\right) \\
& +(2 D-3)\left(f\left(L_{1}+3, L_{i}-1, L_{i+1}+1 ; A_{1}+1\right)-f\left(L_{1}+3, L_{i}-1, L_{i-1}+1 ; A_{1}+1\right)\right\}=0,
\end{aligned}
$$

which is a difference equation in two infinite sets of variables (the $A^{\prime} s$ and the $L^{\prime} s$ ). Based on the exponential dependence on area and length that the Wilson Loop should present in the strong and weak coupling limits, we consider the ansatz

$$
f\left(L_{i} ; A_{i}\right)=\prod_{i=1}^{\infty} X_{i}^{L_{i}} Y_{i}^{A_{i}}
$$

which, when substituted into (33), produces the more amenable system of algebraic equations

$$
(2 D-3) X_{1}^{3} X_{i}^{-1} Y_{1}\left(X_{i+1}-X_{i-1}\right)+X_{1}^{3} X_{i}^{-1} Y_{i}^{-1}\left(X_{i+1} Y_{i+1}-X_{i-1} Y_{i-1}\right)+\frac{i}{\beta}=0, \quad i=1,2, \cdots,
$$

with $X_{0}=Y_{0}=1$. Equation (35) can be seen (for each $i$ ) as a single equation for two unknown functions: $X_{i}$ and $Y_{i}$. Yet, it is not simple enough for our purposes. Hence, we try separately with length-independent and area-independent solutions. In the first case, we set $X_{i}=1 \forall i$, which yields

$$
\beta\left\{Y_{i+1}-Y_{i-1}\right\}+i Y_{i}=0 .
$$

Taking into account the condition $y_{0}=1$, the solution to this equation is

$$
Y_{i}(2 \beta)=\frac{a I_{i}(2 \beta)+b(-1)^{i} K_{i}(2 \beta)}{a I_{0}(2 \beta)+b K_{0}(2 \beta)},
$$


where $I_{i}$ and $K_{i}$ are the modified Bessel functions and $a, b$ are arbitrary constants. Since $Y_{i}(2 \beta)$ must be regular in the strong-coupling limit $(\beta=0)$, we must have $b=0$; hence

$$
Y_{i}=\frac{I_{i}(2 \beta)}{I_{0}(2 \beta)}
$$

On the other hand, the area-independent solution corresponds to setting $Y_{i}=1 \forall i$. This leads to the equation

$$
(D-1) \beta X_{1}^{3}\left(X_{i+1}-X_{i-1}\right)+i X_{i}=0 .
$$

As before, the admissible solution is

$$
X_{i}=\frac{I_{i}\left(4(D-1) \beta X_{1}^{3}\right)}{I_{0}\left(4(D-1) \beta X_{1}^{3}\right)},
$$

that must be understood as follows. For $i=1$, eq. (40) gives a transcendental equation for $X_{1}$. Once this is solved, eq. (40) again allows us to calculate the remaining $X^{\prime} s$.

We recognize that the "area solution" (38) is the exact solution of the $D=2$ theory [26], whereas the "length solution" (40) corresponds to the mean-field solution (which is dimension-dependent), with $X_{1}$ being the "mean link" 27, 28]. In the first case, one obtains a dimension independent result that, although being the exact solution only for $D=2$, it is also a better approximation for the Wilson functional than the mean-field solution in the strong coupling regime, even when $D>2$. In fact, the strong-coupling mean-field solution is $X_{1}=0$, which corresponds to a vanishing Wilson loop. Instead, the "area solution" (38) behaves as $\langle W(C)\rangle=(\beta)^{A}$ for $\beta \rightarrow 0$, which is precisely what results from strong-coupling expansions in any dimension. On the other hand, it is well known that the mean-field approximation provides a qualitatively acceptable description of the weak coupling region for large enough dimensions, although it incorrectly predicts phase transitions for $D=2$ and $D=3$.

Summarizing, we have sketched an approach for studying lattice loop-equations that is based on previous work in the Hamiltonian formulation. This approach, while being relatively simple, could capture some relevant features among those that are responsible for the critical behavior of lattice gauge models. A natural question at this point is whether or not there is a simple extension of these ideas to the non-Abelian models, where loop-equations are considerably more involved. This and other relevant questions are under work.

This work was supported by DID-USB grant GID-30 and Fonacit grant G-2001000712.

[1] R. Gambini and J. Pullin, Loops, Knots, Gauge Theories and Quantum Gravity (Cambridge University Press, Cambridge, UK, (1996)).

[2] C. Rovelli and P. Upadhya, gr-qc/9806079

[3] C. Rovelli, L. Smolin, Nuc. Phys. B 331, 80 (1990).

[4] R. Gambini and A. Trias, Phys. Rev. D 22, 1380 (1980); D 23, 553 (1981); D 27, 2935 (1983); Nucl. Phys. B 278, 436 (1986).

[5] C. Di Bartolo, F. Nori, R. Gambini and A. Trías, Lett. Nuovo Cim. 38, 497 (1983).

[6] C. Rovelli and L. Smolin, Nucl. Phys. B 331, 80 (1990).

[7] R. Gambini, Phys. Lett. B 255, 180 (1991).

[8] C.Rovelli, Living Rev.Rel.1:1 (1998) e-Print Archive: gr-qc/9710008

[9] L.Smolin, hep-th/0303185

[10] T.Thiemann, Lect.Notes Phys.631:41-135 (2003).

[11] R. Gambini and A. Trias, Phys. Rev. Lett. 53, 2359 (1984).

[12] R. Gambini, L. Leal and A. Trias, Phys. Rev. D 39, 3127 (1989).

[13] C. Di Bartolo, R. Gambini and A. Trias, Phys. Rev. D 39, 3136 (1989).

[14] R. Gianvittorio, R. Gambini and A. Trias, Phys. Rev. D 38, 702 (1988).

[15] A.M.Polyakov, Nucl. Phys. B164, 171 (1979).

[16] Yu.M.Makeenko and A.A. Migdal, Phys. Lett. 88B, 135 (1979); 97B, 253 (1980).

[17] Yu.M.Makeenko and A.A. Migdal, Nucl. Phys. B188, 269 (1981).

[18] A.A.Migdal, Phys. Rep.102:4 (1983).

[19] A.A. Migdal, Ann. of Phys. 126, 279 (1980).

[20] G.'t Hooft, Nucl.Phys.B72, 461 (1974).

[21] F.Jugeau and H. Sazdjian, Nucl.Phys.B670:221-263, (2003).

[22] M.Baldicchi and G.M. Prosperi, Phys. Rev.D62, 114024 (2000); D 66, 074008 (2002).

[23] N.Brambilla and a. Vairo, hep-ph/9904330 
[24] She-sheng Xue, Ting-chang Hsien and Chi-min Wu, Phys. Lett.B175, 341 (1986).

[25] G.Bhanot and M.Creutz, Phys. Rev. D21, 2892 (1980).

[26] J.B.Kogut, Rev. of Mod. Phys. 51, 659 (1979).

[27] J-M.Drouffe and J-B.Zuber, Phys. Rep.102:1 (1983).

[28] M.Creutz, "Quarks, Gluons and Lattices", Cambridge University Press (1983).

[29] J. Camacaro, R. Gaitan and L. Leal, Mod. Phys. Lett. A 12, 3081 (1997).

[30] M.Peskin and D. Schröder, "An Introduction to Quantum Field Theory", Springer (1995).

[31] A.Polyakov, Mod. Phys. Lett. A3, 325 (1988).

[32] S. Deser, R.Jackiw and S. Templeton, Ann. Phys. 140, 372 (1982). 